\title{
Derivation of a temperature-dependent accommodation coefficient for use in modeling laser-induced incandescence of soot
}

\author{
H.A. Michelsen
}

Received: 26 June 2008 / Revised version: 9 October 2008 / Published online: 11 November 2008

(C) The Author(s) 2008. This article is published with open access at Springerlink.com

\begin{abstract}
This paper presents a derivation of an expression to estimate the accommodation coefficient for gas collisions with a graphite surface, which is meant for use in models of laser-induced incandescence (LII) of soot. Energy transfer between gas molecules and solid surfaces has been studied extensively, and a considerable amount is known about the physical mechanisms important in thermal accommodation. Values of accommodation coefficients currently used in LII models are temperature independent and are based on a small subset of information available in the literature. The expression derived in this study is based on published data from state-to-state gas-surface scattering experiments. The present study compiles data on the temperature dependence of translational, rotational, and vibrational energy transfer for diatomic molecules (predominantly NO) colliding with graphite surfaces. The data were used to infer partial accommodation coefficients for translational, rotational, and vibrational degrees of freedom, which were consolidated to derive an overall accommodation coefficient that accounts for accommodation of all degrees of freedom of the scattered gas distributions. This accommodation coefficient can be used to calculate conductive cooling rates following laser heating of soot particles.
\end{abstract}

PACS 65.80.+n $\cdot 78.20 . \mathrm{Nv} \cdot 42.62 .-\mathrm{b} \cdot 44.05 .+\mathrm{e}$

H.A. Michelsen ( $\bowtie)$

Combustion Research Facility, Sandia National Labs, Livermore,

CA, USA

e-mail: hamiche@ sandia.gov

Fax: +1-925-2942276

\section{Introduction}

Laser-induced incandescence (LII) has become a popular technique for non-invasive measurements of soot concentration and primary particle size for a variety of applications. This technique involves heating particles with a high-power pulsed laser and collecting the radiative emission from the hot particles. The intensity of the signal depends on the particle volume fraction, making LII a useful tool for measuring soot spatial and temporal distributions under a wide range of conditions [1-3].

The signal decay rate is predominantly controlled by the conductive cooling rate at low fluences and atmospheric pressure. The conductive cooling rate, in turn, depends on the particle surface area to mass ratio. The correlation between this ratio and signal decay rate has been used extensively to infer primary particle size [2, 3]. Quantitative determination of particle size requires a detailed understanding of the physical mechanisms that control the LII signal decay rate and particle cooling rate. A thorough description of the conductive cooling rate, in particular, is required for deriving accurate particle sizes from signal decay rates.

Considerable effort has been devoted to developing models capable of predicting LII signals in response to pulsedlaser heating over a range of fluences [4]. Current models solve the energy- and mass-balance equations to predict the temporal response of the particle to a single laser shot. LII models typically account for particle heating by laser absorption and cooling by conduction to surrounding gases, sublimation of carbon clusters, and emission of thermal radiation. Nevertheless, there are severe limitations in the fundamental understanding of LII signal generation and significant uncertainties in model predictions and measurement interpretations. 
Large uncertainties are associated with the calculated conductive cooling rate. The rate of heat loss by conductive cooling is generally calculated under free-molecular flow conditions according to the expression [4]

$Q_{\text {Cond }}=\frac{\pi D^{2} Z_{\text {surf }}}{N_{a}} \alpha_{T}\left(C_{V}+\frac{R}{2}\right)\left(T_{S}-T_{g}\right)$

or an equivalent expression, where $D$ is the primary particle diameter, $Z_{\text {surf }}$ is the collision rate of the gas with the particle in units of $\mathrm{s}^{-1} \mathrm{~cm}^{-2}, N_{a}$ is the Avogadro constant, $C_{V}$ is the heat capacity of the gas at constant volume, $R$ is the universal gas constant, $T_{S}$ is the surface temperature, $T_{g}$ is the gas temperature, and $\alpha_{T}$ is the thermal accommodation coefficient. The most significant uncertainties in this expression originate from the thermal accommodation coefficient, which is a measure of the energy transferred between a gas molecule and a surface during a molecule-surface interaction. The value of this parameter ranges from 0 (when gassurface collisions are completely elastic) to 1 (when molecules interact with the surface for a sufficiently long time to become fully energetically equilibrated with the surface before leaving it). For lack of better information, current LII models use temperature-independent values for $\alpha_{T}$, despite expectations that this parameter should be dependent on surface temperature and gas temperature [5, 6]. Some LII models use values of 0.07 [7], 0.18 [8], 0.25 [9], 0.37 [10, 11], and 1.0 [12] inferred from measured LII decay rates by fitting model predictions of signal decay rates to measured values and allowing $\alpha_{T}$ to be one of the adjustable parameters. LII models, however, have significant uncertainties beyond the accommodation coefficient, and the conditions for the measurements are difficult to control, making this approach indirect and unreliable. Other models use a value $(0.3)$ based on the measured accommodation coefficient of $\mathrm{N}_{2}$ interacting with a graphite surface at gas temperatures near room temperature and surface temperatures in the range of 1,220$1,270 \mathrm{~K}[13]$. In this experiment rotational temperature gradients were measured as the room-temperature gas interacted with the hot surface; rotational and translational temperatures were assumed to be equilibrated with each other. Although graphite is a good surrogate for soot, and $\mathrm{N}_{2}$ is a good surrogate for air, extrapolating this result to more relevant temperatures (gas temperatures of $\sim 1,700-1,900 \mathrm{~K}$ for flames and surface temperatures of $\sim 2,500-4,000 \mathrm{~K}$ ) is not possible without additional information. This paper attempts to address this problem using information from molecular beam scattering measurements of molecules (particularly diatomic molecules, predominantly NO) on graphite surfaces.

Energy can be transferred between the molecule and the surface via coupling between the translational, rotational, and vibrational degrees of freedom of the molecule and surface phonons. The surface can also facilitate coupling between the degrees of freedom of the molecule striking it.
There is a large body of literature presenting experimental and theoretical studies of internal and kinetic energy transfer between surfaces and gas molecules. Many of these studies focus on the accommodation and coupling of the translational, rotational, and vibrational degrees of freedom of molecules as they interact with the surface. These studies can be extremely helpful in understanding thermal accommodation in general. The extensive state-to-state measurements of NO scattered from a graphite surface provided by Häger, Walther, and coworkers [14-21] in particular are useful for predicting the behavior of the accommodation coefficient under a wide range of conditions. Despite the large number of experimental and theoretical studies of energy transfer between gas molecules and surfaces, this data set is the only one available for scattering from graphite surfaces that is complete enough to derive an accommodation coefficient and extrapolate it to different surface- and gastemperature regimes.

This paper presents a derivation of an expression that accounts for the temperature dependence of the thermal accommodation of individual degrees of freedom of molecules colliding with the surface of a soot particle. The derivation is based on state-to-state gas-surface scattering data available in the literature for diatomic molecules (particularly NO) impinging on a graphite surface. The final expression allows surface temperature and gas temperature to be taken into account in estimating the accommodation coefficient to be used in LII models.

\section{Method}

Molecular beam studies have demonstrated that at low surface temperatures molecules or atoms impinging on a surface will often scatter into two distinct populations. One of these populations is representative of direct inelastic scattering that is not fully accommodated with the surface, and the other is characteristic of trapping-desorption in which molecules are physisorbed to a surface for sufficiently long that internal and kinetic energy of the molecule is more fully equilibrated with the surface. Molecules from a beam undergoing direct inelastic scattering tend to have a narrow velocity distribution in a near-specular direction, whereas molecules undergoing trapping-desorption have a broader, angularly diffuse distribution [22, 23].

Partitioning of scattered molecules into separate diffuse and quasi-specular populations has been observed for tertbutyl chloride [24], fluoroform $\left(\mathrm{CHF}_{3}\right)$ [24], $\mathrm{N}_{2}$ [25], and NO [14-16, 23] scattered from graphite. At higher surface temperatures for which trapping probabilities are low, only the direct inelastic component is observed, resulting in a decrease in thermal accommodation with increasing surface temperature. This behavior has been observed for tert-butyl 
chloride [24], $\mathrm{CHF}_{3}$ [24], $\mathrm{Ar}$ [16], and NO [14-16] scattered from graphite. The diffuse component decreases rapidly between 200 and $730 \mathrm{~K}$ for tert-butyl chloride [24] and $\mathrm{CHF}_{3}$ [24] and disappears at surface temperatures above $\sim 400 \mathrm{~K}$ for $\operatorname{Ar}[16]$ and NO [14-16, 26, 27]. For NO scattered from graphite, the sticking probability has been shown to decrease to zero at about the same surface temperature at which the diffuse component disappears [28].

For surface temperatures at which only direct inelastic scattering is observed, translational accommodation coefficients $\left(\alpha_{\text {Trans }}\right)$ can be derived from beam energies and energy distributions of the direct inelastically scattered molecules using the definition for the accommodation coefficient, i.e.,

$\alpha_{\text {Trans }}=\frac{E_{i}-E_{f}}{E_{i}-2 k_{B} T_{S}}$,

where $E_{i}$ is the kinetic (i.e., translational) energy of the incident beam, $E_{f}$ is the average translational energy of the scattered molecules, $k_{B}$ is the Boltzmann constant, and $T_{S}$ is the surface temperature $[5,29,30]$. Likewise, accommodation coefficients for vibrational $\left(\alpha_{\mathrm{Vib}}\right)$ or rotational $\left(\alpha_{\text {Rot }}\right)$ degrees of freedom can be inferred from internal state distributions of the incident and scattered molecules using a modification of (2), i.e.,

$\alpha_{\mathrm{Vib}, \mathrm{Rot}}=\frac{T_{i}^{\mathrm{Vib}, \mathrm{Rot}}-T_{f}^{\mathrm{Vib}, \mathrm{Rot}}}{T_{i}^{\mathrm{Vib}, \mathrm{Rot}}-T_{S}}$,

where $T_{i}$ is the effective vibrational or rotational temperature of the incident beam, and $T_{f}$ is the effective vibrational or rotational temperature of the scattered molecules $[5,29$, 30].

If the dataset for partial accommodation coefficients is sufficient, we can estimate an overall accommodation coefficient using the expression [31]

$$
\begin{aligned}
\alpha_{T}(U)\left(C_{V}+\frac{R}{2}\right)= & \alpha_{\operatorname{Trans}}\left(C_{V}^{\operatorname{Trans}}+\frac{R}{2}\right) \\
& +\alpha_{\mathrm{Rot}} C_{V}^{\mathrm{Rot}}+\alpha_{\mathrm{Vib}} C_{V}^{\mathrm{Vib}},
\end{aligned}
$$

where $C_{V}^{\text {Trans }}$ is the translational heat capacity of the impinging gas at constant volume, $C_{V}^{\mathrm{Rot}}$ is the corresponding rotational heat capacity, and $C_{V}^{\mathrm{Vib}}$ is the corresponding vibrational heat capacity. For open systems in which pressure is constant and enthalpy $H$ (rather than energy $U$ ) is the parameter of interest [32], (4) can be written with respect to heat capacities at constant pressure, i.e.,

$$
\begin{aligned}
\alpha_{T}(H)\left(C_{P}+\frac{R}{2}\right)= & \alpha_{\operatorname{Trans}}\left(C_{P}^{\mathrm{Trans}}+\frac{R}{2}\right) \\
& +\alpha_{\mathrm{Rot}} C_{P}^{\mathrm{Rot}}+\alpha_{\mathrm{Vib}} C_{P}^{\mathrm{Vib}} .
\end{aligned}
$$

The only difference between (4) and (5) is that the translational accommodation coefficient is weighted slightly more heavily in (5) because $C_{P}^{\text {Trans }}$ is larger than $C_{V}^{\text {Trans }}$ by $R$. These heat capacities can be approximated according to

$C_{V}^{\text {Trans }}=\frac{3}{2} R$,

$C_{P}^{\text {Trans }}=C_{V}^{\text {Trans }}+R=\frac{5}{2} R$,

$C_{P}^{\mathrm{Rot}}=C_{V}^{\mathrm{Rot}}=R$,

$C_{P}^{\mathrm{Vib}}=C_{V}^{\mathrm{Vib}}=R\left(\frac{\Theta_{\mathrm{Vib}}}{T}\right)^{2} \frac{\exp \left(\frac{-\Theta_{\mathrm{Vib}}}{T}\right)}{\left[1-\exp \left(\frac{-\Theta_{\mathrm{Vib}}}{T}\right)\right]^{2}}$,

where the effective "vibrational temperature" is given by [33]

$\Theta_{\mathrm{Vib}}=\frac{\Delta E_{\mathrm{Vib}}}{k_{B}}$,

where $\Delta E_{\mathrm{Vib}}$ is the vibrational energy spacing $\left(1,880 \mathrm{~cm}^{-1}\right)$ [15]. The overall thermal accommodation coefficient can thus be derived from $\alpha_{\text {Trans }}, \alpha_{\text {Rot }}$, and $\alpha_{\mathrm{Vib}}$ using

$\alpha_{T}(H)=\frac{3 \alpha_{\mathrm{Trans}}+\alpha_{\mathrm{Rot}}+\alpha_{\mathrm{Vib}} \frac{C_{P}^{\mathrm{Vib}}}{R}}{4+\frac{C_{P}^{\mathrm{Vib}}}{R}}$

or

$\alpha_{T}(U)=\frac{2 \alpha_{\text {Trans }}+\alpha_{\mathrm{Rot}}+\alpha_{\mathrm{Vib}} \frac{C_{V}^{\mathrm{Vib}}}{R}}{3+\frac{C_{V}^{\mathrm{Vib}}}{R}}$.

\section{Results and discussion}

3.1 Dependence of $\alpha_{T}$ on surface temperature

\subsubsection{Dependence of $\alpha_{\mathrm{Rot}}$ on surface temperature}

Within experimental error the rotational accommodation coefficient for $\mathrm{NO}$ is unity at low surface temperatures of $\leq 300 \mathrm{~K}$, i.e., temperatures at which molecules are predominantly diffusively scattered [14]. This observation is independent of initial rotational temperature and surface temperature. Figure 1a shows the rotational temperature of the scattered molecules as a function of surface temperature for beams with initial rotational temperatures of 20 and $35 \mathrm{~K}$, first excited and ground-state vibrational states, incidence energies of 87.5 and $284 \mathrm{meV}$, and incidence angles of $30^{\circ}, 60^{\circ}$, and $70^{\circ}$. The data are from Häger, Walther, and coworkers $[15,17,18,20,21]$. The data presented in Fig. 1a demonstrate that $\alpha_{\text {Rot }}$ is independent of vibrational state and incidence angle. Whereas at low surface 


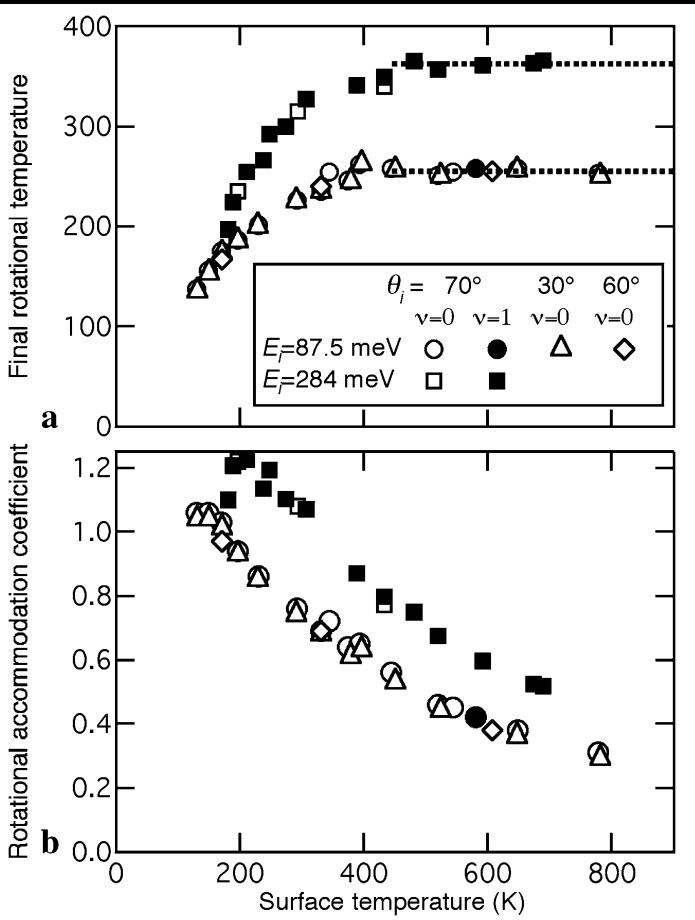

Fig. 1 Surface-temperature dependence of the rotational temperature and associated rotational accommodation coefficient of NO scattered from a graphite surface. (a) Final rotational temperatures and (b) rotational accommodation coefficients are shown as a function of surface temperature for several incidence angles, incidence energies, and initial vibrational states (see legend). Squares and circles represent values from Häger, Walther, and coworkers [20, 21], and triangles and diamonds signify values from Häger and Walther [17, 18]. The values are given in Table 1. The dotted lines in (a) represent the average value of measurements recorded at surface temperatures $>400 \mathrm{~K}$ for each incidence energy

temperatures the scattered molecules are rotationally fully accommodated with the surface ( $\left.T_{f}^{\mathrm{Rot}} \approx T_{S}\right)$, at higher surface temperatures $T_{f}^{\text {Rot }}$ is independent of $T_{S}$. This behavior has been reproduced using classical trajectory methods [34] and a statistical model approach [35]. Both simulation techniques suggest that it results from the conservation of the component of angular momentum normal to the surface. In the present study this behavior is used to extrapolate the results to higher surface temperatures, i.e., the final rotational temperature is assumed to be independent of $T_{S}$ at surface temperatures higher than those measured, as indicated by the dotted lines in Fig. 1a. In the limit of high surface temperature this behavior yields an inverse surface temperature dependence for the rotational accommodation coefficient, i.e., $\alpha_{\text {Rot }} \propto 1 / T_{S}$ for large $T_{S}$. Figure $1 \mathrm{~b}$ and Table 1 present values of $\alpha_{\text {Rot }}$ derived from the data shown in Fig. 1a. These data were from Fig. 2 of Vach et al. [20], Fig. 8 of Häger and Walther [21], and Fig. 5 of Häger and Walther $[17,18]$.

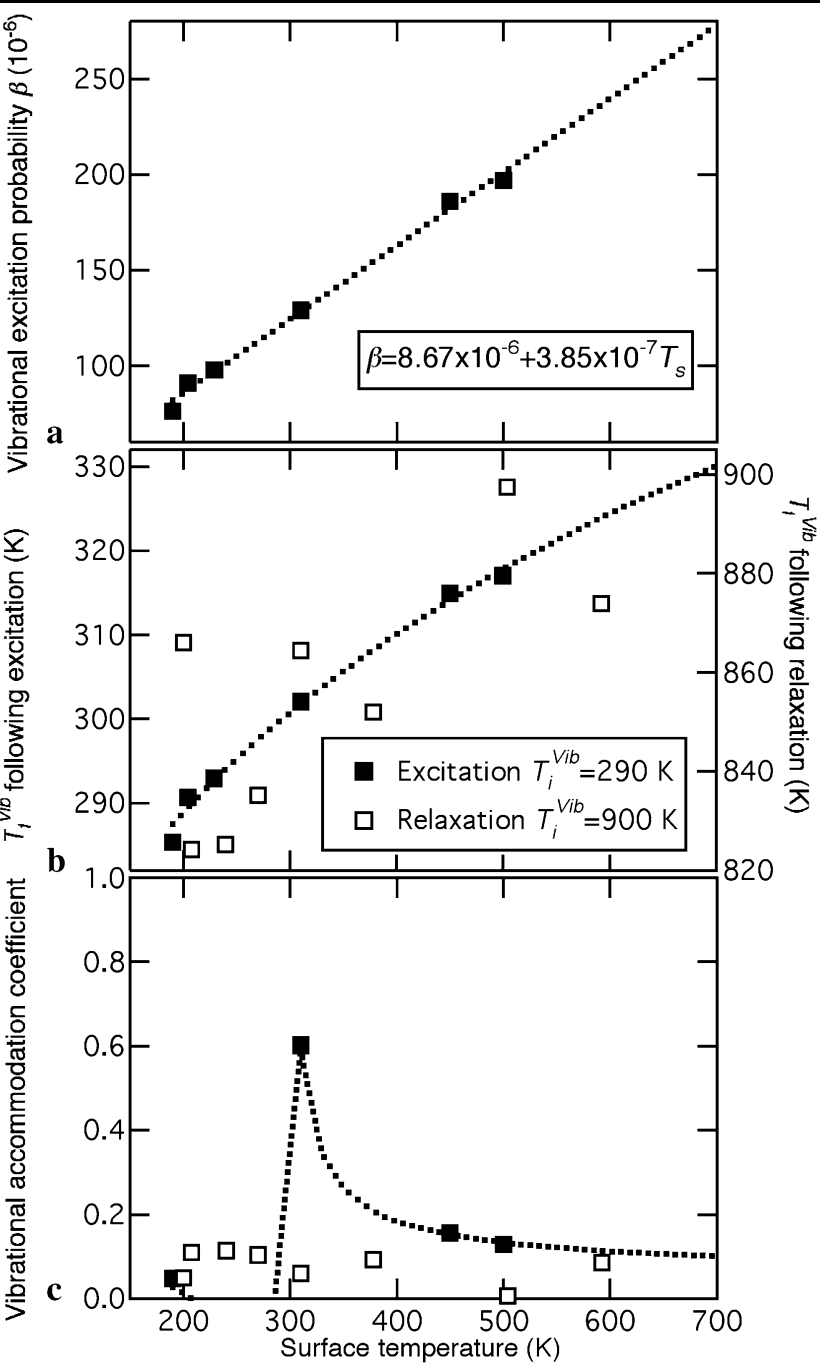

Fig. 2 Surface-temperature dependence of vibrational energy transfer for NO scattered from a graphite surface. (a) Vibrational excitation probabilities, (b) final vibrational temperatures, and (c) vibrational accommodation coefficients are shown as a function of surface temperature. The solid squares represent values for vibrational excitation of molecules with an initial vibrational temperature of $290 \mathrm{~K}$ from Vach et al. [15], and open squares represent values for vibrational relaxation of molecules with an initial vibrational temperature of $900 \mathrm{~K}$ from Vach et al. [20]. These values are given in Table 2. All data were recorded with an incidence energy of $284 \mathrm{meV}$ and incidence angle of $70^{\circ}$. The dotted lines represent a linear fit to the vibrational excitation probability data in (a) and the corresponding (b) final vibrational temperature and (c) vibrational accommodation coefficient

\subsubsection{Dependence of $\alpha_{\mathrm{Vib}}$ on surface temperature}

Figure 2a shows the vibrational excitation probability of an NO molecule in its vibrational ground state scattered from a graphite surface. The data are from Fig. 8 of Vach et al. [15] for a beam of $\mathrm{NO}$ with an initial vibrational temperature of $\sim 290 \mathrm{~K}$, incidence energy of $284 \mathrm{meV}$, and incidence angle 
Table 1 Rotational

accommodation coefficients

inferred from gas-surface

scattering measurements on

graphite over a range of surface

temperatures

\begin{tabular}{|c|c|c|c|c|c|c|c|}
\hline Species & $T_{S}(\mathrm{~K})$ & $T_{i}^{\mathrm{Rot}}(\mathrm{K})$ & $T_{f}^{\text {Rot }}(\mathrm{K})$ & $E_{i}(\mathrm{meV})$ & $T_{g}(\mathrm{~K})$ & $\alpha_{\text {Rot }}$ & Reference \\
\hline \multirow[t]{3}{*}{$\mathrm{NO}$} & 196 & 20 & 235 & 284 & 1,650 & 1.22 & \multirow{3}{*}{$\begin{array}{l}\text { Fig. } 2 \text { of }[20] \\
\text { Fig. } 8 \text { of }[21] \\
(v=0) \rightarrow(v=0)\end{array}$} \\
\hline & 293 & 20 & 315 & 284 & 1,650 & 1.08 & \\
\hline & 433 & 20 & 340 & 284 & 1,650 & 0.77 & \\
\hline \multirow[t]{14}{*}{$\mathrm{NO}$} & 181 & 20 & 197 & 284 & 1,650 & 1.10 & \multirow{26}{*}{$\begin{array}{l}\text { Fig. } 2 \text { of }[20] \\
\text { Fig. } 8 \text { of }[21] \\
(v=1) \rightarrow(v=1) \\
\theta_{i}=70^{\circ}\end{array}$} \\
\hline & 189 & 20 & 224 & 284 & 1,650 & 1.21 & \\
\hline & 211 & 20 & 254 & 284 & 1,650 & 1.23 & \\
\hline & 237 & 20 & 266 & 284 & 1,650 & 1.13 & \\
\hline & 248 & 20 & 292 & 284 & 1,650 & 1.19 & \\
\hline & 274 & 20 & 300 & 284 & 1,650 & 1.10 & \\
\hline & 307 & 20 & 327 & 284 & 1,650 & 1.07 & \\
\hline & 389 & 20 & 341 & 284 & 1,650 & 0.87 & \\
\hline & 433 & 20 & 349 & 284 & 1,650 & 0.80 & \\
\hline & 481 & 20 & 365 & 284 & 1,650 & 0.75 & \\
\hline & 519 & 20 & 357 & 284 & 1,650 & 0.68 & \\
\hline & 592 & 20 & 361 & 284 & 1,650 & 0.60 & \\
\hline & 674 & 20 & 363 & 284 & 1,650 & 0.52 & \\
\hline & 689 & 20 & 366 & 284 & 1,650 & 0.52 & \\
\hline \multirow[t]{12}{*}{ Extrapolated values } & 1,000 & 20 & 362 & 284 & 1,650 & 0.35 & \\
\hline & 1,200 & 20 & 362 & 284 & 1,650 & 0.29 & \\
\hline & 1,400 & 20 & 362 & 284 & 1,650 & 0.25 & \\
\hline & 1,600 & 20 & 362 & 284 & 1,650 & 0.22 & \\
\hline & 1,800 & 20 & 362 & 284 & 1,650 & 0.19 & \\
\hline & 2,000 & 20 & 362 & 284 & 1,650 & 0.17 & \\
\hline & 2,500 & 20 & 362 & 284 & 1,650 & 0.14 & \\
\hline & 3,000 & 20 & 362 & 284 & 1,650 & 0.11 & \\
\hline & 3,500 & 20 & 362 & 284 & 1,650 & 0.10 & \\
\hline & 4,000 & 20 & 362 & 284 & 1,650 & 0.09 & \\
\hline & 4,500 & 20 & 362 & 284 & 1,650 & 0.08 & \\
\hline & 5,000 & 20 & 362 & 284 & 1,650 & 0.07 & \\
\hline NO & 581 & 20 & 258 & 87.5 & 508 & 0.42 & \\
\hline \multirow[t]{3}{*}{ NO } & 196 & 20 & 235 & 284 & 1,650 & 1.22 & \multirow{18}{*}{$\begin{array}{l}\text { Fig. } 2 \text { of }[20] \\
\text { Fig. } 8 \text { of }[21] \\
(v=0) \rightarrow(v=0) \\
\theta_{i}=70^{\circ}\end{array}$} \\
\hline & 293 & 20 & 315 & 284 & 1,650 & 1.08 & \\
\hline & 433 & 20 & 340 & 284 & 1,650 & 0.77 & \\
\hline \multirow[t]{15}{*}{ NO } & 130 & 20 & 137 & 87.5 & 508 & 1.06 & \\
\hline & 148 & 20 & 156 & 87.5 & 508 & 1.06 & \\
\hline & 170 & 20 & 175 & 87.5 & 508 & 1.03 & \\
\hline & 196 & 20 & 186 & 87.5 & 508 & 0.94 & \\
\hline & 230 & 20 & 201 & 87.5 & 508 & 0.86 & \\
\hline & 292 & 20 & 226 & 87.5 & 508 & 0.76 & \\
\hline & 330 & 20 & 235 & 87.5 & 508 & 0.69 & \\
\hline & 344 & 20 & 254 & 87.5 & 508 & 0.72 & \\
\hline & 374 & 20 & 254 & 87.5 & 508 & 0.64 & \\
\hline & 393 & 20 & 246 & 87.5 & 508 & 0.65 & \\
\hline & 444 & 20 & 262 & 87.5 & 508 & 0.56 & \\
\hline & 519 & 20 & 258 & 87.5 & 508 & 0.46 & \\
\hline & 544 & 20 & 251 & 87.5 & 508 & 0.45 & \\
\hline & 648 & 20 & 258 & 87.5 & 508 & 0.38 & \\
\hline & 778 & 20 & 253 & 87.5 & 508 & 0.31 & \\
\hline
\end{tabular}




\begin{tabular}{|c|c|c|c|c|c|c|c|c|}
\hline Table 1 (Continued) & Species & $T_{S}(\mathrm{~K})$ & $T_{i}^{\mathrm{Rot}}(\mathrm{K})$ & $T_{f}^{\mathrm{Rot}}(\mathrm{K})$ & $E_{i}(\mathrm{meV})$ & $T_{g}(\mathrm{~K})$ & $\alpha_{\text {Rot }}$ & Reference \\
\hline & \multirow[t]{13}{*}{ NO } & 131 & 35 & 135 & 87.5 & 508 & 1.04 & \multirow{13}{*}{$\begin{array}{l}\text { Fig. } 5 \text { of }[17,18] \\
(v=0) \rightarrow(v=0) \\
\theta_{i}=30^{\circ}\end{array}$} \\
\hline & & 149 & 35 & 153 & 87.5 & 508 & 1.04 & \\
\hline & & 171 & 35 & 173 & 87.5 & 508 & 1.01 & \\
\hline & & 196 & 35 & 185 & 87.5 & 508 & 0.93 & \\
\hline & & 229 & 35 & 200 & 87.5 & 508 & 0.85 & \\
\hline & & 291 & 35 & 225 & 87.5 & 508 & 0.74 & \\
\hline & & 331 & 35 & 235 & 87.5 & 508 & 0.68 & \\
\hline & & 378 & 35 & 244 & 87.5 & 508 & 0.61 & \\
\hline & & 396 & 35 & 262 & 87.5 & 508 & 0.63 & \\
\hline & & 451 & 35 & 256 & 87.5 & 508 & 0.53 & \\
\hline & & 524 & 35 & 250 & 87.5 & 508 & 0.44 & \\
\hline & & 647 & 35 & 256 & 87.5 & 508 & 0.36 & \\
\hline & & 782 & 35 & 250 & 87.5 & 508 & 0.29 & \\
\hline & \multirow[t]{3}{*}{ NO } & 171 & 35 & 167 & 87.5 & 508 & 0.97 & Fig. 5 of $[17,18]$ \\
\hline & & 331 & 35 & 240 & 87.5 & 508 & 0.69 & $(v=0) \rightarrow(v=0)$ \\
\hline & & 607 & 35 & 255 & 87.5 & 508 & 0.38 & $\theta_{i}=60^{\circ}$ \\
\hline
\end{tabular}

of $70^{\circ}$. The excitation probability is defined as

$\beta=\frac{N(v=0 \rightarrow v=1)}{N(v=0)}$,

where $N(v=0)$ represents the population of the vibrational ground state, and $N(v=0 \rightarrow v=1)$ represents the number of molecules excited from the ground state to the first excited vibrational state. This excitation probability is associated with a final vibrational temperature according to [15]

$\beta=\exp \left(\frac{-\Delta E_{\mathrm{Vib}}}{k_{B} T_{f}^{\mathrm{Vib}}}\right)$.

The excitation probability is linearly dependent on surface temperature over the surface temperature measured. The data were extrapolated to higher surface temperatures by fitting the data in Fig. 2a and assuming a linear dependence at higher temperatures. Figure $2 b$ shows the surfacetemperature dependence of the final vibrational temperature estimated by rearranging (14), which gives

$T_{f}^{\mathrm{Vib}}=\frac{-\Delta E_{\mathrm{Vib}}}{k_{B} \ln \beta}$.

The dotted line is inferred from the linear fit to the data in Fig. 2a. Figure $2 \mathrm{~b}$ also presents values of $T_{f}^{\mathrm{Vib}}$ derived from survival probabilities of vibrationally excited NO scattered from a graphite surface. Figure 3 of Vach et al. [20] provides survival probabilities of NO $(v=1)$ in a beam with $5 \%$ vibrationally excited molecules also with an incidence energy of $284 \mathrm{meV}$ and incidence angle of $70^{\circ}$. Multiplying these survival probabilities by 0.05 yields the population of vibrationally excited molecules scattered from the surface, the equivalent of $\beta$ from which $T_{f}^{\mathrm{Vib}}$ is calculated. The values derived using these relaxation observations demonstrate considerably more scatter than those based on excitation experiments. The vibrational accommodation coefficients derived from these data are presented in Fig. $2 \mathrm{c}$ and Table 2. In contrast to the behavior shown for $\alpha_{\text {Rot }}$ in Fig. $1 b, \alpha_{\mathrm{Vib}}$ decreases very gradually with increasing surface temperature. The large change near $300 \mathrm{~K}$ is related to the proximity of the surface temperature to the initial vibrational temperature of $290 \mathrm{~K}$ for the excitation experiments. Under such conditions, the accommodation coefficient is undefined.

Vibrational accommodation would be expected to be higher for larger molecular species, which have more vibrational modes and smaller vibrational spacings. This prediction is supported by values of $\alpha_{\mathrm{Vib}}$ for $\mathrm{CF}_{3} \mathrm{Br}$ scattered from graphite compared to extrapolated values for NO shown in Fig. 3. These values are also given in Table 2. They were derived from the results of Petterson and coworkers [36, 37] and demonstrate that the vibrational accommodation coefficient for $\mathrm{CF}_{3} \mathrm{Br} /$ graphite decreases substantially with increasing surface temperature at surface temperatures of $500-1,200 \mathrm{~K}$.

Andersson et al. [38] have noted that the vibrational temperature of $\mathrm{SF}_{6}$ scattered from graphite demonstrates no dependence on surface temperature at high surface temperatures $(950-1,400 \mathrm{~K})$. This observation implies a decrease in the vibrational accommodation coefficient with increasing surface temperature, as shown in Fig. 3 and Table 2. Despite the large number of vibrational degrees of freedom, values of $\alpha_{\mathrm{Vib}}$ derived from these data [38] are comparable to the extrapolated values for $\mathrm{NO}$ and significantly lower than those for $\mathrm{CF}_{3} \mathrm{Br}$ at similar surface tem- 
Table 2 Vibrational

accommodation coefficients inferred from gas-surface

scattering measurements on graphite over a range of surface temperatures

\begin{tabular}{|c|c|c|c|c|c|c|c|}
\hline Species & $T_{S}(\mathrm{~K})$ & $T_{i}^{\mathrm{Vib}}(\mathrm{K})$ & $T_{f}^{\mathrm{Vib}}(\mathrm{K})$ & $E_{i}(\mathrm{meV})$ & $T_{g}(\mathrm{~K})$ & $\alpha_{\mathrm{Vib}}$ & Reference \\
\hline \multirow[t]{8}{*}{ NO } & 200 & 900 & 866 & 284 & 1,650 & 0.049 & \multirow[t]{8}{*}{ Fig. 3 of [20] } \\
\hline & 208 & 900 & 824 & 284 & 1,650 & 0.110 & \\
\hline & 240 & 900 & 825 & 284 & 1,650 & 0.113 & \\
\hline & 270 & 900 & 835 & 284 & 1,650 & 0.103 & \\
\hline & 310 & 900 & 864 & 284 & 1,650 & 0.060 & \\
\hline & 378 & 900 & 852 & 284 & 1,650 & 0.092 & \\
\hline & 504 & 900 & 898 & 284 & 1,650 & 0.006 & \\
\hline & 592 & 900 & 874 & 284 & 1,650 & 0.085 & \\
\hline \multirow[t]{6}{*}{ NO } & 190 & 290 & 285 & 284 & 1,650 & 0.047 & \multirow[t]{20}{*}{ Fig. 8 of [15] } \\
\hline & 204 & 290 & 291 & 284 & 1,650 & -0.008 & \\
\hline & 229 & 290 & 293 & 284 & 1,650 & -0.048 & \\
\hline & 310 & 290 & 302 & 284 & 1,650 & 0.602 & \\
\hline & 450 & 290 & 315 & 284 & 1,650 & 0.156 & \\
\hline & 500 & 290 & 317 & 284 & 1,650 & 0.129 & \\
\hline \multirow[t]{14}{*}{ Extrapolated values } & 600 & 290 & 324 & 284 & 1,650 & 0.111 & \\
\hline & 800 & 290 & 336 & 284 & 1,650 & 0.090 & \\
\hline & 1,000 & 290 & 345 & 284 & 1,650 & 0.078 & \\
\hline & 1,200 & 290 & 353 & 284 & 1,650 & 0.069 & \\
\hline & 1,400 & 290 & 360 & 284 & 1,650 & 0.063 & \\
\hline & 1,600 & 290 & 367 & 284 & 1,650 & 0.058 & \\
\hline & 1,800 & 290 & 373 & 284 & 1,650 & 0.055 & \\
\hline & 2,000 & 290 & 378 & 284 & 1,650 & 0.051 & \\
\hline & 2,500 & 290 & 390 & 284 & 1,650 & 0.045 & \\
\hline & 3,000 & 290 & 400 & 284 & 1,650 & 0.041 & \\
\hline & 3,500 & 290 & 410 & 284 & 1,650 & 0.037 & \\
\hline & 4,000 & 290 & 418 & 284 & 1,650 & 0.035 & \\
\hline & 4,500 & 290 & 426 & 284 & 1,650 & 0.032 & \\
\hline & 5,000 & 290 & 433 & 284 & 1,650 & 0.030 & \\
\hline \multirow[t]{2}{*}{$\mathrm{CF}_{3} \mathrm{Br}$} & 830 & 320 & 427 & 690 & 4000 & 0.21 & \multirow[t]{2}{*}{ Table 1 of [36] } \\
\hline & 1,170 & 320 & 466 & 690 & 4000 & 0.17 & \\
\hline \multirow[t]{5}{*}{$\mathrm{CF}_{3} \mathrm{Br}$} & 500 & 300 & 405 & 1600 & 9280 & 0.53 & \multirow[t]{5}{*}{ Fig. 6 of [37] } \\
\hline & 600 & 300 & 400 & 1600 & 9280 & 0.33 & \\
\hline & 800 & 300 & 440 & 1600 & 9280 & 0.28 & \\
\hline & 1,000 & 300 & 475 & 1600 & 9280 & 0.25 & \\
\hline & 1,200 & 300 & 500 & 1600 & 9280 & 0.22 & \\
\hline \multirow[t]{3}{*}{$\mathrm{SF}_{6}$} & 950 & 300 & 318 & 640 & 3710 & 0.028 & \multirow{6}{*}{$\begin{array}{l}\text { Fig. } 2 \text { of [38] } \\
\text { assuming } T_{f} \text { not } \\
\text { dependent on } T_{S}\end{array}$} \\
\hline & 1,170 & 300 & 318 & 640 & 3710 & 0.021 & \\
\hline & 1,400 & 300 & 318 & 640 & 3710 & 0.016 & \\
\hline \multirow[t]{3}{*}{$\mathrm{SF}_{6}$} & 950 & 300 & 346 & 1690 & 9810 & 0.071 & \\
\hline & 1,170 & 300 & 346 & 1690 & 9810 & 0.053 & \\
\hline & 1,400 & 300 & 346 & 1690 & 9810 & 0.042 & \\
\hline
\end{tabular}

peratures and incidence energies. A study by van Opbergen et al. [39] has shown that at lower surface temperatures $(178-425 \mathrm{~K})$ the vibrational temperature of scat- tered $\mathrm{SF}_{6}$ increases significantly with surface temperature [39], but their paper does not provide sufficient information to determine whether the vibrational accommodation 


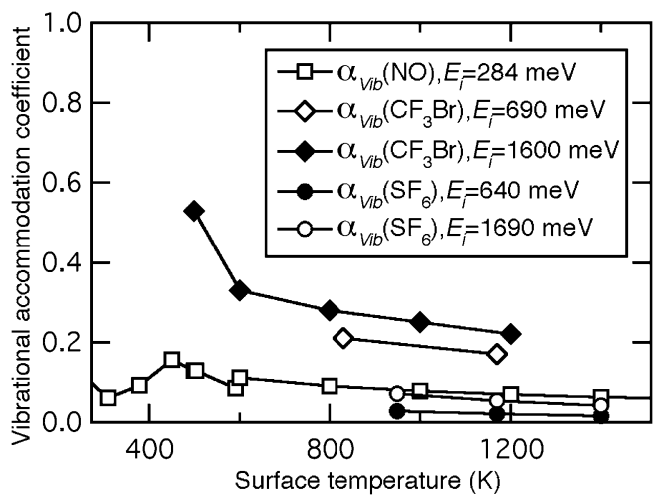

Fig. 3 Surface-temperature dependence of the vibrational accommodation coefficient for $\mathrm{NO}, \mathrm{CF}_{3} \mathrm{Br}$, and $\mathrm{SF}_{6}$ scattered from a graphite surface. The squares represent values derived and extrapolated from measurements of Vach et al. [15] recorded with an incidence angle of $70^{\circ}$. Values for $\mathrm{CF}_{3} \mathrm{Br}$ were derived from data from Andersson et al. [36] (open diamonds) and Någård et al. [37] (solid diamonds) recorded with an incidence angle of $45^{\circ}$. Values for $\mathrm{SF}_{6}$ were derived from data from Andersson et al. [38] (circles) recorded with an incidence angle of $21^{\circ}$. Incidence energies are given in the legend. Values of accommodation coefficients are given in Table 2

coefficient increases or decreases with surface temperature.

\subsubsection{Dependence of $\alpha_{\text {Trans }}$ on surface temperature}

Inferring translational accommodation coefficients from the available surface scattering data is more complicated than determining either rotational or vibrational accommodation coefficients. The extent of translational accommodation depends on incidence angle, which must be taken into account when determining the integrated accommodation coefficient. Translational accommodation also depends on scattering angle, which must be accounted for in the analysis when data are not collected at the peak of the angular distribution.

Figure 4a presents data from Fig. 6 of Vach et al. [21], which shows the peak of the time-of-flight velocity distribution as a function of surface temperature (open triangles). The data were measured with an incidence angle of $50^{\circ}$, a fixed scattering angle of $45^{\circ}$, and an incidence velocity of $1,350 \mathrm{~m} / \mathrm{s}$, which corresponds to an incidence energy of $284 \mathrm{meV}$. Figure 5 presents data from a number of studies $[14,16-19,21]$ showing that the peak of the angular distribution of the scattered molecules decreases with increasing surface temperature. The data shown in Fig. 4a were recorded at an angle smaller than the peak of the scattered angular distribution. In addition, the velocity of the scattered molecules decreases with increasing scattering angle $[15,16,20]$. Hence, the measured velocities shown in Fig. $4 \mathrm{a}$ are higher than the most probable velocities for the scattered distributions.

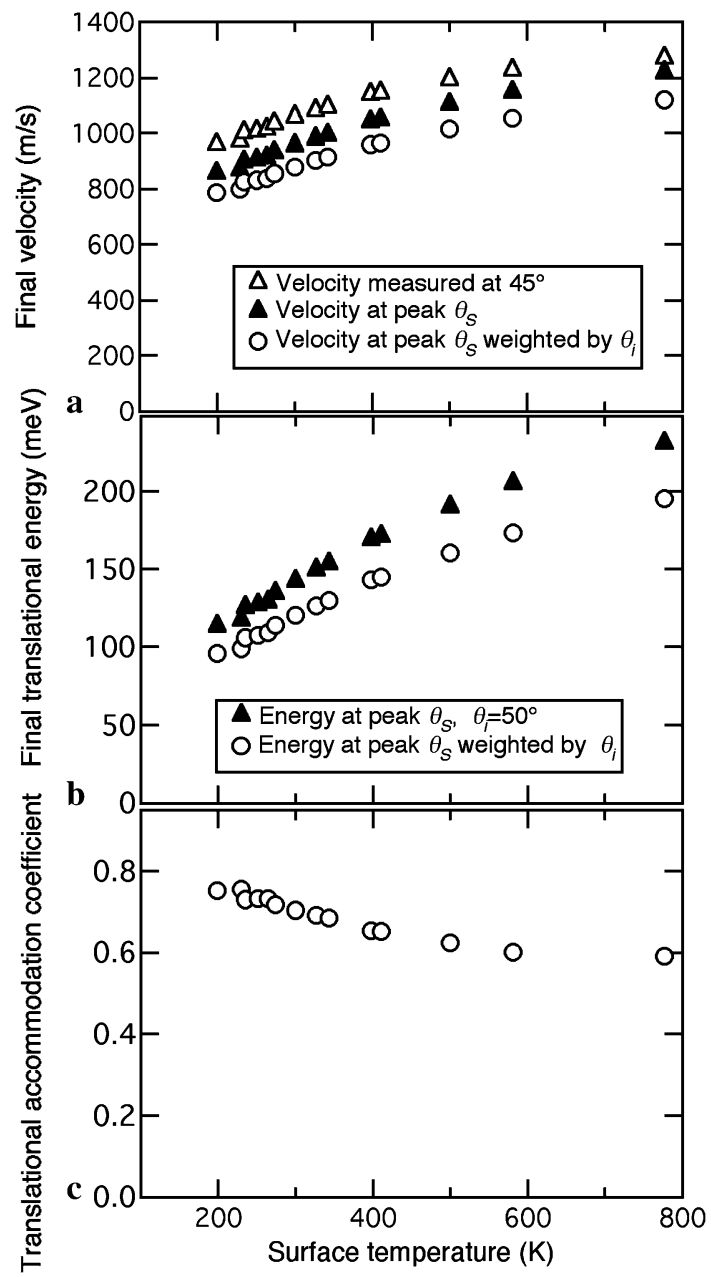

Fig. 4 Surface-temperature dependence of the (a) final velocity, (b) final translational energy, and (c) translational accommodation coefficient for NO scattered from a graphite surface (given in Table 3). The open triangles in (a) represent measurements of Vach et al. [15] recorded with an incidence angle of $50^{\circ}$ and scattering angle of $45^{\circ}$ with an incidence energy of $284 \mathrm{meV}$. The solid triangles in (a) and (b) show these measurements corrected to represent values at the peak scattering angle. The circles in each panel show the results corresponding to the initial measurements corrected for peak scattering angle and averaged over all incidence angles

The most probable velocity at a particular surface temperature can be estimated by first identifying the peak scattering angle at this surface temperature and then determining the velocity at this angle. Figure 5 shows that linear fits to several data sets of peak scattering angle vs $T_{S}$ yield the same slope of $-0.0175 \mathrm{deg} / \mathrm{K}$. The peak scattering angle at each surface temperature can be derived for the data in Fig. 4a using this slope scaled to a value of $56^{\circ}$, the peak angle at $500 \mathrm{~K}$ derived from the data in Fig. 5 of Vach et al. [15]. The result is represented by the dotted curve in Fig. 5. Figure 6 shows the ratio of the velocity at the peak of the angular distribution relative to the velocity at some scattering angle as a function of the difference between that scattering angle and the peak angle. The 


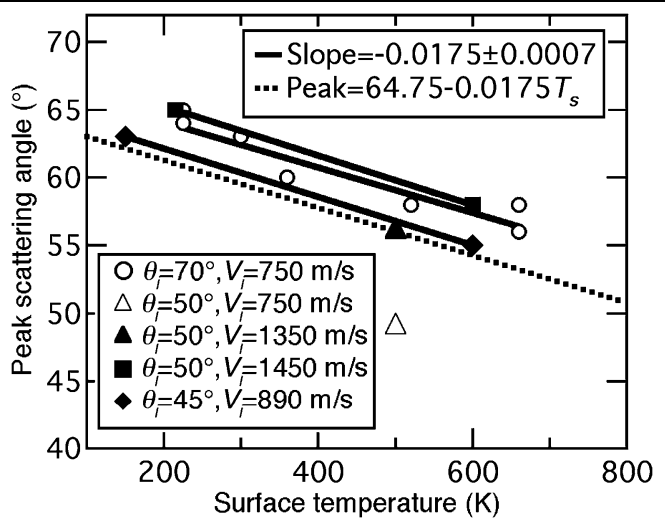

Fig. 5 Surface-temperature dependence of the peak scattering angle for NO scattered from a graphite surface. The symbols represent values from Häger et al. [16, 18] (circles), Häger and Walther [17] (open triangle), Vach et al. [15] (solid triangle), Kuze et al. [19] (squares), and Häger et al. [14] (diamonds). The solid lines represent linear fits to the data sets with more than one point. The dotted line represents a line with the slope given by the average of the three fits (solid lines), adjusted to pass through the solid triangle

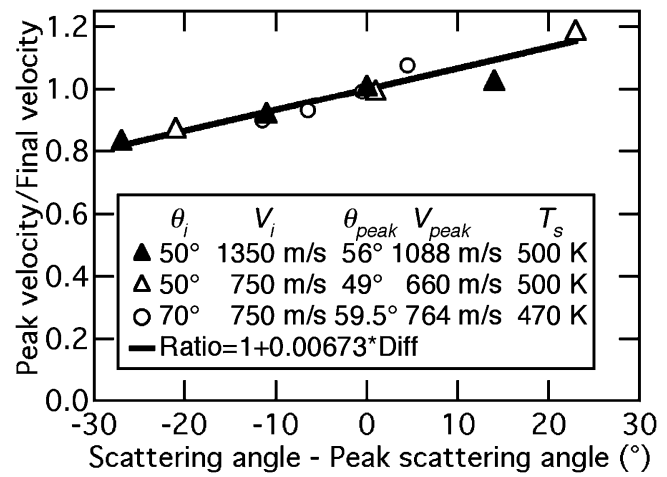

Fig. 6 Velocity at the peak of the angular distribution relative to the measured velocity as a function of the angular difference between the scattering angle at which the measurements were made and the peak of the angular distribution. The symbols represent values from Häger et al. [16] (circles), Häger and Walther [17] and Vach et al. [15] (open triangles), and Vach et al. [15] (solid triangles). The solid line represents a linear fit to the data sets combined

results are scaled to unity when the scattering angle is at the peak of the angular distribution. The results from several experiments fall along the same line with a slope of $0.00673 / \mathrm{deg}$. The values of the peak scattering angle derived as a function of surface temperature in Fig. 5 were subtracted from the scattering angle $\left(45^{\circ}\right)$. This difference was then used to determine the ratio of the peak velocity relative to velocities at other angles. The measured velocities shown in Fig. 4a were multiplied by this ratio to convert the measured velocities to velocities at the peak scattering angle, and the results are compared to the original values in Fig. 4a. The velocities at the peak of the angular distribution were converted to energies and are displayed in Fig. 4b.

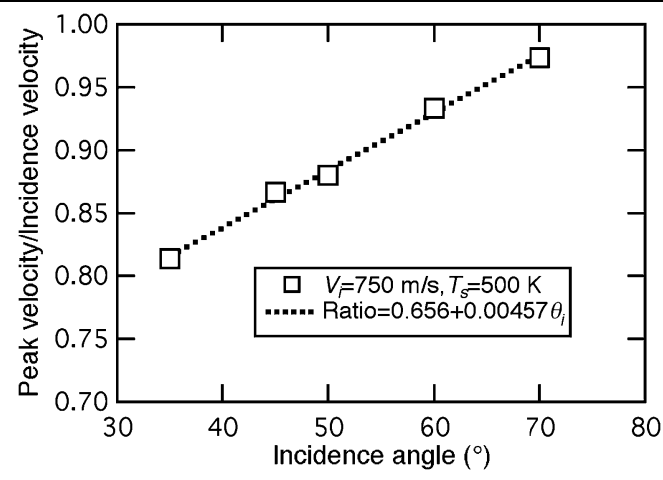

Fig. 7 Velocity at the peak of the angular distribution relative to the incidence velocity as a function of the incidence angle. The symbols represent values from Häger and Walther [17]. The dotted line represents a linear fit to the data

Because momentum parallel to the surface is more likely to be conserved during the gas-surface collision than momentum perpendicular to the surface, the incidence angle must also be taken into account in determining accommodation. Figure 7 shows a linear increase of the most probable velocity with increasing incidence angle. These results are scaled by the incidence energy and were derived from measurements of Häger and Walther [17]. Performing a linear fit to the data in Fig. 7 gives an intercept of 0.656 and a slope of $0.00457 /$ degree or $0.262 /$ radian. An average over incidence angle can be derived according to

$$
\begin{aligned}
\left\langle\frac{V_{\text {peak }}}{V_{i}}\right\rangle & =\frac{\int_{0}^{\pi / 2}\left(0.656+0.262 \theta_{i}\right) \cos \theta_{i} d \theta_{i}}{\int_{0}^{\pi / 2} \cos \theta_{i} d \theta_{i}} \\
& =0.8055 .
\end{aligned}
$$

Dividing this average value by 0.88 , the value for $50^{\circ}$ (the incidence angle for the data in Fig. 4), gives a multiplicative correction factor of 0.92 for the dependence of the final velocity on incidence angle over all solid angles. The results are shown in Fig. 4a and presented as final kinetic energies in Fig. 4b. Figure 4c and Table 3 present the corresponding translational accommodation coefficients.

Previous work has suggested that the average final kinetic energy is linearly dependent on both incidence energy and surface temperature [40, 41], i.e.,

$E_{f}=a_{1} E_{i}+a_{2} E_{S}$

where $E_{S}=2 k_{B} T_{S}$, and $a_{1}$ and $a_{2}$ are constants. Values of $E_{f}$ shown in Fig. 4b, however, are not fit very well by this functional form. In this case $E_{i}$ is constant $(284 \mathrm{meV})$, and $E_{f}$ appears to demonstrate saturation behavior at high surface temperatures. Figure $8 \mathrm{a}$ presents the results of fits using functions that follow the form of (17) in the limit of low surface temperature and start to saturate at high surface tem- 
Table 3 Translational accommodation coefficients inferred from gas-surface scattering measurements on graphite over a range of surface temperatures

\begin{tabular}{|c|c|c|c|c|c|c|c|}
\hline Species & $T_{S}(\mathrm{~K})$ & $E_{S}(\mathrm{meV})$ & $E_{f}(\mathrm{meV})$ & $E_{i}(\mathrm{meV})$ & $T_{g}(\mathrm{~K})$ & $\alpha_{\text {Trans }}$ & Reference \\
\hline \multirow[t]{14}{*}{ NO } & 199 & 34 & 96 & 284 & 1,650 & 0.75 & \multirow[t]{26}{*}{ Fig. 6 of [15] } \\
\hline & 230 & 40 & 99 & 284 & 1,650 & 0.76 & \\
\hline & 235 & 41 & 106 & 284 & 1,650 & 0.73 & \\
\hline & 251 & 43 & 108 & 284 & 1,650 & 0.73 & \\
\hline & 264 & 45 & 109 & 284 & 1,650 & 0.73 & \\
\hline & 274 & 47 & 114 & 284 & 1,650 & 0.72 & \\
\hline & 300 & 52 & 120 & 284 & 1,650 & 0.70 & \\
\hline & 327 & 56 & 127 & 284 & 1,650 & 0.69 & \\
\hline & 343 & 59 & 130 & 284 & 1,650 & 0.69 & \\
\hline & 398 & 69 & 143 & 284 & 1,650 & 0.65 & \\
\hline & 411 & 71 & 145 & 284 & 1,650 & 0.65 & \\
\hline & 500 & 86 & 161 & 284 & 1,650 & 0.62 & \\
\hline & 581 & 100 & 174 & 284 & 1,650 & 0.60 & \\
\hline & 777 & 134 & 195 & 284 & 1,650 & 0.59 & \\
\hline \multirow[t]{12}{*}{ Extrapolated values } & 1,000 & 172 & 228 & 284 & 1,650 & 0.50 & \\
\hline & 1,200 & 207 & 248 & 284 & 1,650 & 0.47 & \\
\hline & 1,400 & 241 & 265 & 284 & 1,650 & 0.43 & \\
\hline & 1,600 & 276 & 280 & 284 & 1,650 & 0.39 & \\
\hline & 1,800 & 310 & 292 & 284 & 1,650 & 0.36 & \\
\hline & 2,000 & 345 & 304 & 284 & 1,650 & 0.32 & \\
\hline & 2,500 & 431 & 327 & 284 & 1,650 & 0.29 & \\
\hline & 3,000 & 517 & 344 & 284 & 1,650 & 0.26 & \\
\hline & 3,500 & 603 & 358 & 284 & 1,650 & 0.23 & \\
\hline & 4,000 & 689 & 369 & 284 & 1,650 & 0.21 & \\
\hline & 4,500 & 776 & 379 & 284 & 1,650 & 0.19 & \\
\hline & 5,000 & 862 & 387 & 284 & 1,650 & 0.18 & \\
\hline $\mathrm{N}_{2}$ & 1,245 & 215 & & & 293 & 0.26 & [13] \\
\hline
\end{tabular}

perature. These functions are:

Error function $E_{f}=a_{1} E_{i}+a_{2} a_{3} \operatorname{erf}\left(\frac{E_{S}}{a_{3}}\right)$;

Exponential function

$$
E_{f}=a_{1} E_{i}+a_{2} a_{3}\left[1-\exp \left(\frac{-E_{S}}{a_{3}}\right)\right]
$$

Ratio $E_{f}=a_{1} E_{i}+a_{2}\left(\frac{E_{S}}{1+\frac{E_{S}}{a_{3}}}\right)$,

where $a_{3}$ is a constant. For these equations,

$$
\begin{aligned}
& \lim _{E_{S} \rightarrow 0} E_{f}=a_{1} E_{i}+a_{2} E_{S}, \\
& \lim _{E_{S} \rightarrow \infty} E_{f}=a_{1} E_{i}+a_{2} a_{3} .
\end{aligned}
$$

The functions were fit to the $\theta_{i}$-weighted data from Fig. $4 \mathrm{~b}$ in addition to a point constrained to $E_{f}=284 \mathrm{meV}$ at $E_{S}=E_{i}=284 \mathrm{meV}$. The best fit is provided by (20), as shown by the values of $\chi^{2}$ for the results given in Table 4 . Figure $8 \mathrm{~b}$ presents the translational accommodation coefficients inferred from these fits compared with the values inferred from the measurements. The linear fit using (17) gives very poor agreement with the values inferred from the measurements, as expected from the poor fit provided by this function. The other fits give much better agreement with the inferred values and extrapolate to much lower values of $\alpha_{\text {Trans }}$ than estimated using the linear function. The deviations from a smooth curve near $T_{S}=1,650 \mathrm{~K}$ occur at the point where $E_{f} \approx E_{S} \approx E_{i}$. In the results presented below and in Table $3, \alpha_{\text {Trans }}$ is extrapolated to higher surface temperatures using the best-fit function, i.e., (20), and values of $\alpha_{\text {Trans }}$ near $T_{S}=1,650 \mathrm{~K}$ have been interpolated.

The results in Fig. 8 demonstrate a decrease in $\alpha_{\text {Trans }}$ with increasing surface temperature for both the points inferred from the data and the extrapolated values. Other data sets that provide information for $t$-butyl chloride [24], $\mathrm{CHF}_{3}$ [24], and $\mathrm{Xe}$ [42] scattered from graphite as a func- 
Table 4 Results of fits of final kinetic energy $E_{f}$ as a function of $E_{S}=2 k_{B} T_{S}$ for extrapolating $\alpha_{\text {Trans }}$ to higher surface temperatures

${ }^{\mathrm{a}} \chi^{2}=$

$\sum_{j}\left(E_{f, j}^{\text {measured }}-E_{f, j}^{\text {predicted }}\right)^{2}$ for each point $j$

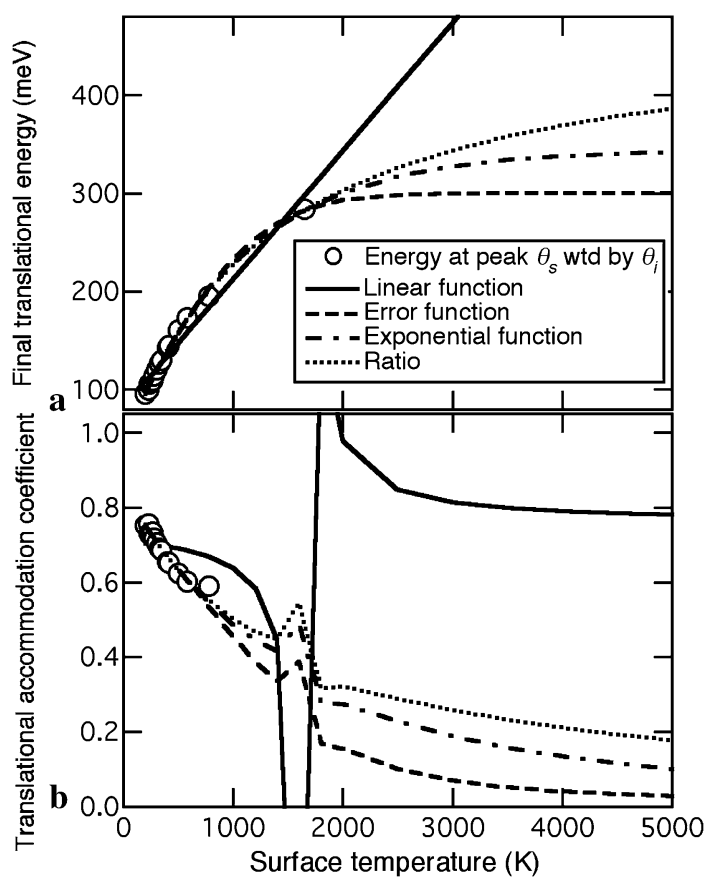

Fig. 8 Surface-temperature dependence of the (a) final translational energy and (b) translational accommodation coefficient for NO scattered from a graphite surface extrapolated to high surface temperatures. The circles in (a) are the same as those in Fig. 4b, and the circles in (b) are the same as those in Fig. $4 \mathrm{c}$ and are derived from measurements of Vach et al. [15]. The lines represent results of fits to the data in (a) using (17) (solid line), (18) (dashed line), (19) (dot-dashed line), and (20) (dotted line). The fit parameters are given in Table 4, and the fits in (a) were used to derived the lines in (b)

tion of surface temperature are not complete enough to derive translational accommodation coefficients.

\subsubsection{Combined dependence of $\alpha_{T}$ on surface temperature}

In general thermal accommodation appears to decrease with increasing surface temperature at surface temperatures above that at which trapping/desorption is significant. The overall thermal accommodation coefficient of $\mathrm{H}_{2}$ on graphite has been observed to increase with increasing surface temperature at low surface temperatures (77-340 K) [43] but decrease with increasing temperature at higher temperatures (from 0.82 at $77 \mathrm{~K}$ to 0.15 at $1,450 \mathrm{~K}$ ) [13, 31, 43 , 44], and those of $\mathrm{Kr}, \mathrm{Xe}$, and $\mathrm{CH}_{4}$ have also been ob-

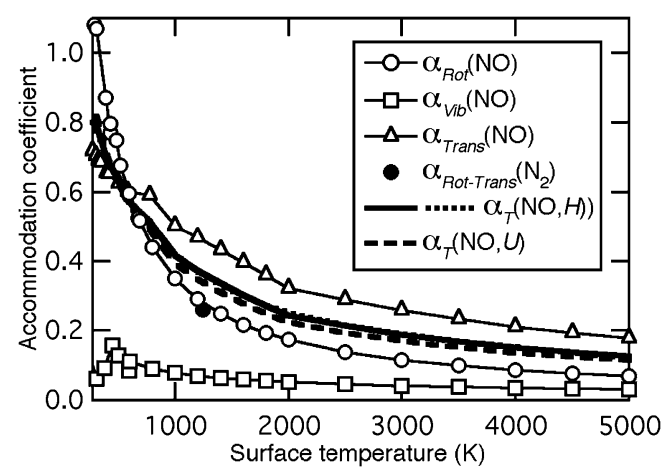

Fig. 9 Surface-temperature dependence of the partial accommodation coefficients for translational, rotational, and vibrational degrees of freedom of NO scattered from a graphite surface. Open symbols represent values derived from measurements from Häger, Walther, and coworkers [14-21] and extrapolated to higher surface temperatures, as described in the text. The solid circle is from Leroy et al. [13] for $\mathrm{N}_{2}$ in which translation and rotation were probably equilibrated with each other via gas-phase collisions; the gas temperature was $293 \mathrm{~K}$. The solid line was derived using (11), and the dashed line was derived using (12). The dotted line (barely visible) is a fit to the solid line. Values of the points shown are given in Tables 1-3

served to decrease with increasing surface temperature from $850-1,400 \mathrm{~K}$ [31].

Figure 9 shows a comparison of values of rotational, vibrational, and translational accommodation coefficients for NO scattered from graphite extrapolated to high surface temperatures, as described above. The coupling between translational and rotational degrees of freedom is generally strong $[22,35]$, and translational and rotational accommodation coefficients are often assumed to be comparable. Figure 9 suggests that rotational accommodation is higher at low surface temperatures and lower at high surface temperatures than translational accommodation. Studies of $\mathrm{NO} /$ graphite scattering have shown very similar angular, rotational, and translational distributions for molecules scattering to and from different vibrational states, which indicates that rotational and translational degrees of freedom are decoupled from vibrational degrees of freedom for this system [15]. The results of this study suggested that all degrees of freedom were strongly coupled to surface phonons. In general vibrational accommodation tends to be significantly lower than accommodation of translational and rotational degrees of freedom, particularly for molecules with large vibrational spacings, which is consistent with the re- 


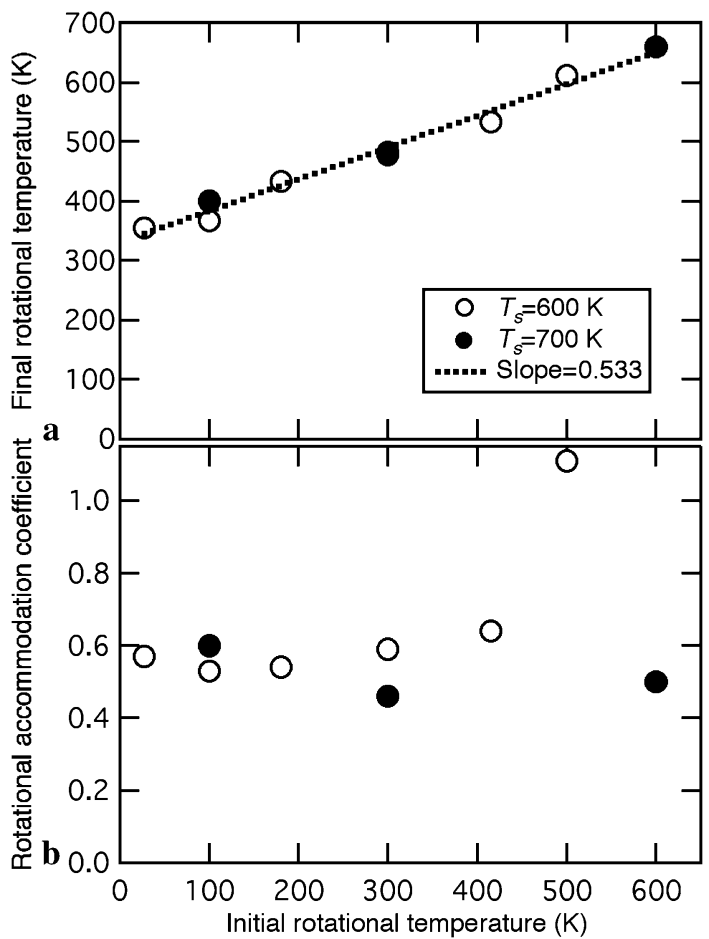

Fig. 10 Dependence of the (a) final rotational temperature and (b) rotational accommodation coefficient on initial rotational temperature of NO scattered from a graphite surface. The symbols in (a) represent data from Häger et al. [14], from which the points in (b) were derived. The data were recorded with an incidence energy of $124 \mathrm{meV}$ and incidence angle of $45^{\circ}$ at surface temperatures of 600 and $700 \mathrm{~K}$ as indicated in the legend. The dotted line in (a) shows a linear fit to the data

sults shown in Fig. 9. Surface roughness will also have an effect. The surface roughness of diamond(111) or sputtered carbon is much higher than for graphite, trapping is more effective, and accommodation coefficients are larger [29, 45].

Equation (11) or (12) can be used to derive an overall accommodation coefficient as a function of surface temperature from these data. The results of these calculations are also shown in Fig. 9. An exponential decay with a sloping baseline (dotted line) does a good job of fitting the curve derived from (11) (solid line) and yields

$$
\begin{aligned}
\alpha_{T}\left(H, T_{S}\right)= & {\left[0.28-3.23 \times 10^{-5} T_{S}\right.} \\
& \left.+0.800 \exp \left(-1.53 \times 10^{-3} T_{S}\right)\right] .
\end{aligned}
$$

The data used to derive this equation were recorded at an effective gas temperature $\left(T_{g}=E_{i} / 2 k_{B}\right)$ of $1,650 \mathrm{~K}$.

\subsection{Dependence of $\alpha_{T}$ on gas temperature}

\subsubsection{Dependence of $\alpha_{\mathrm{Rot}}$ on gas temperature}

Because $T_{f}^{\text {Rot }}$ depends on neither initial vibrational state [20, $21]$ nor incidence angle [17, 18], only the incidence energy and initial rotational temperature need to be considered in

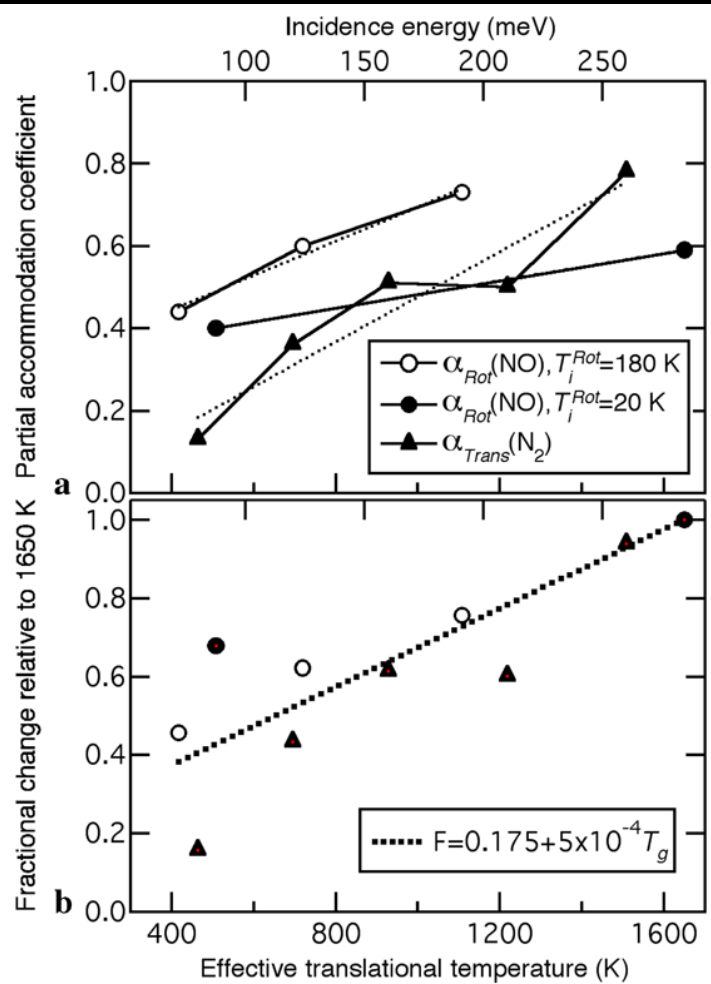

Fig. 11 Effective gas-temperature dependence of the accommodation coefficients for translational and rotational degrees of freedom of NO and $\mathrm{N}_{2}$ scattered from a graphite surface. Circles represent values derived from measurements of $\mathrm{NO}$ at a surface temperature of $600 \mathrm{~K}$ from Häger et al. [14] for an incidence angle of $45^{\circ}$ and initial rotational temperature of $180 \mathrm{~K}$ (open circles) and from Häger et al. [20, 21] for an incidence angle of $70^{\circ}$ and initial rotational temperature of $20 \mathrm{~K}$ (solid circles). Solid triangles represent values derived from measurements of $\mathrm{N}_{2}$ at a surface temperature of $293 \mathrm{~K}$ and incidence angle of $60^{\circ}$ from Kinefuchi et al. [25]. (a) Partial accommodation coefficients are shown as a function of effective translational temperature, which is assumed to be associated with the gas temperature according to $T_{g} \approx T_{i}^{\text {Trans }} \equiv E_{i} / 2 k_{B}$. These values are given in Tables 5 (circles) and 7 (triangles). The dotted lines show linear fits to each data set. (b) Values from (a) were scaled to unity at 1,650 K using the predicted values at $1,650 \mathrm{~K}$ from the linear fits. The dotted line shows a fit to the combined scaled data set to derive a gas-temperature scaling factor

determining the effect of gas temperature on the final rotational temperature and rotational accommodation coefficient. Figure 1 shows that, at high surface temperatures, $T_{f}^{\text {Rot }}$ and $\alpha_{\text {Rot }}$ depend on incidence energy, which is associated with a translational temperature of the incoming gas molecules. The final rotational temperature also depends on the initial rotational temperature. Figure 10a shows that, for a fixed incidence energy, $T_{f}^{\text {Rot }}$ increases linearly with $T_{i}^{\text {Rot }}$. A linear fit to the data yields a slope of 0.533 . Figure $10 \mathrm{~b}$ shows the corresponding rotational accommodation coefficient as a function of initial rotational temperature. Although $T_{f}^{\mathrm{Rot}}$ increases linearly with $T_{i}^{\mathrm{Rot}}, \alpha_{\mathrm{Rot}}$ is nearly independent of $T_{i}^{\mathrm{Rot}}$ at higher surface temperatures, as long as the rotational temperature is significantly different from the surface temperature. These plots were derived from data given in 
Figs. 5 and 6 of Häger et al. [14] for an incidence energy of $124 \mathrm{meV}$, incidence angle of $45^{\circ}$, and surface temperatures of 600 and $700 \mathrm{~K}$.

There are fewer data points to use to assess the impact of translational temperature on $\alpha_{\text {Rot }}$. Figure 11a shows $\alpha_{\text {Rot }}$ plotted as a function of the effective translational temperature, which is assumed to correspond to the gas temperature and is approximated from the incidence energy using the expression $T_{g} \approx T_{i}^{\text {Trans }} \approx E_{i} / 2 k_{B}$. Values of $\alpha_{\text {Rot }}$ were derived from data taken from Fig. 4 of Häger et al. [14] and Fig. 8 of Häger and Walther [21] for a surface temperature of $600 \mathrm{~K}$. Values are given in Table 5. The data indicate an increase in $\alpha_{\text {Rot }}$ with increasing $T_{i}^{\text {Trans }}$.

\subsubsection{Dependence of $\alpha_{\mathrm{Vib}}$ on gas temperature}

The results in Fig. 2c demonstrate little dependence of $\alpha_{\mathrm{Vib}}$ on the initial vibrational temperature. In addition, there appears to be very little coupling between molecular vibrational and other molecular degrees of freedom for the $\mathrm{NO}$ /graphite system. The vibrational state of the incident or scattered molecules does not appear to influence the rotational temperature, velocity distribution, or angular distribution of the scattered molecules [15, 20, 21]. The results of Vach et al. $[15,20]$ suggest that vibrational accom- modation proceeds via close coupling between the molecular vibrational degree of freedom and those of surface phonons. These results indicate that the vibrational accommodation coefficient does not depend on gas temperature.

Figure 3 shows $\alpha_{\mathrm{Vib}}$ for $\mathrm{CF}_{3} \mathrm{Br}$ scattered from a graphite surface at two significantly different incidence energies. Even for the $\mathrm{CF}_{3} \mathrm{Br} /$ graphite system, for which exchange of vibrational energy with the surface is relatively efficient, there appears to be little dependence on incidence energy. At much higher incidence energies there appears to be an increase in the vibrational accommodation coefficient with incidence energy for both $\mathrm{CF}_{3} \mathrm{Br}[36,37]$ and $\mathrm{SF}_{6}$ [38] (see Table 6), but these energies correspond to effective translational temperatures exceeding $14,000 \mathrm{~K}$, which is beyond the range of interest for the present study.

\subsubsection{Dependence of $\alpha_{\text {Trans }}$ on gas temperature}

The available data are limited for assessing the effect of gas temperature on $\alpha_{\text {Trans }}$. As shown above, relatively comprehensive data sets have been published for the $\mathrm{NO}$ /graphite system at incidence velocities of 750 and $1,350 \mathrm{~m} / \mathrm{s}$, but most of the data at $750 \mathrm{~m} / \mathrm{s}$ were recorded at a surface
Table 5 Rotational accommodation coefficients inferred from gas-surface scattering measurements on graphite over a range of effective gas temperatures

\begin{tabular}{lllllrll}
\hline Species & $T_{S}(\mathrm{~K})$ & $T_{i}^{\text {Rot }}(\mathrm{K})$ & $T_{f}^{\text {Rot }}(\mathrm{K})$ & $E_{i}(\mathrm{meV})$ & $T_{g}(\mathrm{~K})$ & $\alpha_{\text {Rot }}$ & Reference \\
\hline NO & 600 & 180 & 365 & 72 & 417 & 0.44 & Fig. 4 of [14] \\
& 600 & 180 & 430 & 124 & 719 & 0.60 & \\
& 600 & 180 & 485 & 191 & 1,110 & 0.73 & \\
NO & 600 & 20 & 255 & 87.5 & 508 & 0.40 & Fig. 2 of [20] \\
& 600 & 20 & 362 & 284 & 1,650 & 0.59 & Fig. 8 of [21] \\
\hline
\end{tabular}

\begin{tabular}{lccccccc}
\hline Species & $T_{S}(\mathrm{~K})$ & $T_{i}^{\mathrm{Vib}}(\mathrm{K})$ & $T_{f}^{\mathrm{Vib}}(\mathrm{K})$ & $E_{i}(\mathrm{meV})$ & $T_{g}(\mathrm{~K})$ & $\alpha_{\text {Vib }}$ & Reference \\
\hline $\mathrm{CF}_{3} \mathrm{Br}$ & 830 & 320 & 427 & 690 & 4,000 & 0.21 & Table 1 of [36] \\
& 830 & 320 & 460 & 1,560 & 9,050 & 0.27 & \\
& 830 & 320 & 484 & 2,450 & 14,200 & 0.32 & \\
& & & & & & & \\
$\mathrm{CF}_{3} \mathrm{Br}$ & 830 & 300 & 438 & 500 & 2,900 & 0.26 & Fig. 5 of [37] \\
& 830 & 300 & 430 & 1,500 & 8,700 & 0.24 & \\
& 830 & 300 & 430 & 1,900 & 11,000 & 0.24 & \\
& 830 & 300 & 450 & 2,500 & 14,500 & 0.28 & \\
& 830 & 300 & 510 & 4,000 & 23,200 & 0.40 & \\
$\mathrm{SF}_{6}$ & 830 & 300 & 550 & 5,250 & 30,500 & 0.47 & \\
& 1,170 & 300 & 318 & 640 & 3,710 & 0.021 & Fig. 2 of [38] \\
& 1,170 & 300 & 324 & 1,420 & 8,120 & 0.028 & \\
& 1,170 & 300 & 354 & 2,500 & 14,500 & 0.062 & \\
\hline
\end{tabular}

Table 6 Vibrational accommodation coefficients inferred from gas-surface scattering measurements on graphite over a range of effective gas temperatures 
Table 7 Translational

accommodation coefficients inferred from gas-surface scattering measurements on graphite over a range of effective gas temperatures

\begin{tabular}{lllcrrll}
\hline Species & $T_{S}(\mathrm{~K})$ & $E_{S}(\mathrm{meV})$ & $E_{f}(\mathrm{meV})$ & $E_{i}(\mathrm{meV})$ & $T_{g}(\mathrm{~K})$ & $\alpha_{\text {Trans }}$ & Reference \\
\hline $\mathrm{N}_{2}$ & 293 & 50 & 76 & 80 & 460 & 0.13 & Fig. 5 of [25] \\
& 293 & 50 & 95 & 120 & 700 & 0.36 & $\theta_{i}=60^{\circ}$ \\
& 293 & 50 & 104 & 160 & 930 & 0.51 & \\
& 293 & 50 & 130 & 210 & 1,220 & 0.50 & \\
& 293 & 50 & 96 & 260 & 1,510 & 0.78 & \\
$\mathrm{Xe}$ & 550 & 95 & 238 & 450 & 2,610 & 0.60 & Figs. 2 \& 3 of [42] \\
& 550 & 95 & 766 & 1,560 & 9,050 & 0.54 & Figs. 3 \& 4 of [46] \\
& 550 & 95 & 1,709 & 3,620 & 21,000 & 0.54 & $\theta_{i}=35^{\circ}$ \\
\hline
\end{tabular}

temperature of $500 \mathrm{~K}$, for which $E_{s} \approx E_{i}$ and values of $\alpha_{\text {Trans }}$ are unreliable. There are some data available for $\mathrm{N}_{2}$ scattered from graphite, as shown in Fig. 11a and tabulated in Table 7. This data set is not as complete as that for $\mathrm{NO}$ /graphite, and correction for the effect of incidence angle is not possible. Nevertheless, the data set provides some information about the effect of incidence energy on accommodation at a single incidence angle of $60^{\circ}$ and a surface temperature of $293 \mathrm{~K}$. The results suggest that $\alpha_{\text {Trans }}$ increases with increasing gas temperature for $\mathrm{N}_{2}$ scattered from graphite.

As noted above, measurements of Xe scattered from graphite surfaces are also not complete enough to derive an accommodation coefficient. At a single incidence angle of $35^{\circ}$, however, the translational accommodation of Xe [42, 46] on graphite appears to be approximately independent of incidence translational energy (see Table 7). These data were taken at relatively high incidence energies for which the effective translational temperatures were between 2,600 and $21,000 \mathrm{~K}$.

\subsubsection{Combined dependence of $\alpha_{T}$ on gas temperature}

In general, thermal accommodation tends to increase with increasing incidence translational energy, i.e., gas temperature. The available data sets are not complete enough even for NO/graphite, however, to generate an overall accommodation coefficient as a function of effective gas temperature, such as that generated as a function of surface temperature and shown in Fig. 9. In order to estimate the dependence on gas temperature, each data set in Fig. 11a was fit to a line (dotted lines) and scaled to unity at an effective translational temperature of $1,650 \mathrm{~K}$, as shown in Fig. 11b. The fractional change was inferred by a global linear fit to the scaled data (dotted line in Fig. 11b). The result is a multiplicative factor that lowers the accommodation coefficient at gas temperatures below 1,650 K and increases it above this gas temperature.

\subsection{Grand finale}

Including the gas-temperature correction factor in (23) yields

$$
\begin{aligned}
\alpha_{T}\left(H, T_{S}, T_{g}\right)= & {\left[0.28-3.23 \times 10^{-5} T_{S}\right.} \\
& \left.+0.800 \exp \left(-1.53 \times 10^{-3} T_{S}\right)\right] \\
& \times\left(0.175+5 \times 10^{-4} T_{g}\right) .
\end{aligned}
$$

The corresponding expression for $U$ is

$$
\begin{aligned}
\alpha_{T}\left(U, T_{S}, T_{g}\right)= & {\left[0.28-3.50 \times 10^{-5} T_{S}\right.} \\
& \left.+0.934 \exp \left(-1.82 \times 10^{-3} T_{S}\right)\right] \\
& \times\left(0.175+5 \times 10^{-4} T_{g}\right) .
\end{aligned}
$$

The difference between (24) and (25) is small, as shown in Fig. 9.

Equations (24) and (25) predict a value for $\alpha_{T}$ of 0.18 0.19 for a gas temperature of $1,900 \mathrm{~K}$ and surface temperature of $3,500 \mathrm{~K}$. This value is lower than the range of values (0.23-0.37) often used in LII models, for which these gas and surface temperatures are typical [4].

Validation of this accommodation coefficient would involve comparison with total accommodation coefficient measurements of NO on graphite at defined surface and gas temperatures, which are currently unavailable. Confirmation would also benefit from measurements of other gases with graphite, particularly diatomics and especially $\mathrm{N}_{2}$. Overall accommodation coefficients are available for $\mathrm{H}_{2}$ and $\mathrm{O}_{2}$ $[31,43,44]$, but both of these species tend to dissociate on graphite at higher temperatures.

\section{Conclusions}

State-to-state scattering measurements were used to derive an expression for the overall accommodation coefficient of NO with graphite as a function of surface temperature and 
gas temperature. The resulting expression, when extrapolated to surface and gas temperatures frequently encountered during LII measurements, yields an estimate for the accommodation coefficient of $0.18-0.19$, which is lower than the range of values often used in LII models [4]. Useful measurements for validating and extending this analysis would include (1) state-to-state measurements of NO scattered from graphite at higher surface temperatures and as a function of incidence energy, (2) state-to-state measurements of other gases, particularly $\mathrm{N}_{2}$, scattered from graphite, (3) overall accommodation coefficients of relevant species with graphite under well-defined conditions, and (4) measurements on both single crystal and polycrystalline graphite surfaces.

Acknowledgements I thank Tom Settersten for enlightening discussions about energy transfer. This work was funded by the Division of Chemical Sciences, Geosciences, and Biosciences, the Office of Basic Energy Sciences, the US Department of Energy. Sandia is a multiprogram laboratory operated by Sandia Corporation, a Lockheed Martin Company, for the National Nuclear Security Administration under contract DE-AC04-94-AL85000.

Open Access This article is distributed under the terms of the Creative Commons Attribution Noncommercial License which permits any noncommercial use, distribution, and reproduction in any medium, provided the original author(s) and source are credited.

\section{References}

1. L.A. Melton, Appl. Opt. 23, 2201 (1984)

2. R.J. Santoro, C.R. Shaddix, Laser-induced incandescence, in $A p$ plied Combustion Diagnostics, ed. by K. Kohse-Höinghaus, J.B. Jeffries (Taylor \& Francis, New York, 2002), pp. 252-286

3. C. Schulz, B.F. Kock, M. Hofmann, H.A. Michelsen, S. Will, B. Bougie, R. Suntz, G.J. Smallwood, Appl. Phys. B 83, 333 (2006)

4. H.A. Michelsen, F. Liu, B.F. Kock, H. Bladh, A. Boiarciuc, M. Charwath, T. Dreier, R. Hadef, M. Hofmann, J. Reimann, S. Will, P.-E. Bengtsson, H. Bockhorn, F. Foucher, K.P. Geigle, C. Mounaïm-Rousselle, C. Schulz, R. Stirn, B. Tribalet, R. Suntz, Appl. Phys. B 87, 503 (2007)

5. F.O. Goodman, Prog. Surf. Sci. 5, 261 (1974)

6. F.O. Goodman, H.Y. Wachman, Dynamics of Gas-Surface Scattering (Academic, New York, 1976)

7. T. Lehre, B. Jungfleisch, R. Suntz, H. Bockhorn, Appl. Opt. 42, 2021 (2003)

8. K.J. Daun, G.J. Smallwood, F. Liu, J. Heat Transfer 130, 121201 (2008)

9. S.-A. Kuhlmann, J. Reimann, S. Will, J. Aerosol Sci. 37, 1696 (2006)

10. F. Liu, M. Yang, F.A. Hill, D.R. Snelling, G.J. Smallwood, Appl. Phys. B 83, 383 (2006)

11. D.R. Snelling, F. Liu, G.J. Smallwood, Ö.L. Gülder, Combust. Flame 136, 180 (2004)

12. R. Starke, B. Kock, P. Roth, Shock Waves 12, 351 (2003)
13. O. Leroy, J. Perrin, J. Jolly, M. Péalat, M. Lefebvre, J. Phys. D: Appl. Phys. 30, 499 (1997)

14. J. Häger, D. Glatzer, H. Kuze, M. Fink, H. Walther, Surf. Sci. 374, 181 (1997)

15. H. Vach, J. Häger, H. Walther, J. Chem. Phys. 90, 6701 (1989)

16. J. Häger, Y.R. Shen, H. Walther, Phys. Rev. A 31, 1962 (1985)

17. J. Häger, H. Walther, J. Vac. Sci. Technol. B 3, 1490 (1985)

18. J. Häger, H. Walther, Nucl. Instrum. Methods Phys. Res. Sect. A 239, 425 (1985)

19. H. Kuze, J. Häger, H. Walther, Chem. Phys. Lett. 153, 569 (1988)

20. H. Vach, J. Häger, H. Walther, Chem. Phys. Lett. 133, 279 (1987)

21. J. Häger, H. Walther, Annu. Rev. Mater. Sci. 19, 265 (1989)

22. C.T. Rettner, D.J. Auerbach, J.C. Tully, A.W. Kleyn, J. Phys. Chem. 100, 13021 (1996)

23. F. Pradére, A. De Martino, C. Flytzanis, Proc. Indian Acad. Sci. (Chem. Sci.) 103, 237 (1991)

24. S.I. Ionov, M.E. LaVilla, R.S. Mackay, R.B. Bernstein, J. Chem. Phys. 93, 7406 (1990)

25. I. Kinefuchi, H. Yamagachi, S. Shiozaki, Y. Sakiyama, Y. Matsumoto, Out-of-plane scattering distribution of nitrogen molecular beam on graphite (0001) surface, in Rarefied Gas Dynamics: 24th International Symposium (American Institute of Physics, 2005)

26. F. Frenkel, J. Häger, W. Krieger, H. Walther, G. Ertl, J. Segner, W. Vielhaber, Chem. Phys. Lett. 90, 225 (1982)

27. J. Häger, M. Fink, H. Walther, Surf. Sci. 550, 35 (2004)

28. J. Segner, H. Robota, W. Vielhaber, G. Ertl, F. Frenkel, J. Häger, W. Krieger, H. Walther, Surf. Sci. 131, 273 (1983)

29. C.T. Rettner, IEEE Trans. Magn. 34, 2387 (1998)

30. P.L. Houston, R.P. Merrill, Chem. Rev. 88, 657 (1988)

31. S.C. Saxena, R.K. Joshi, Thermal Accommodation and Absorption Coefficients of Gases (McGraw-Hill, New York, 1981)

32. H.A. Michelsen, M.A. Linne, B.F. Kock, M. Hofmann, B. Tribalet, C. Schulz, Appl. Phys. B 93, 645 (2008)

33. R.S. Berry, S.A. Rice, J. Ross, Physical Chemistry (Wiley, New York, 1980)

34. J.B.C. Pettersson, G. Nyman, L. Holmlid, J. Chem. Phys. 89, 6963 (1988)

35. G. Nyman, L. Holmlid, J.B.C. Pettersson, J. Chem. Phys. 93, 845 (1990)

36. M.B. Andersson, J.B.C. Pettersson, N. Marković, Surf. Sci. 384, L880 (1997)

37. M.B. Någård, N. Marković, J.B.C. Pettersson, J. Chem. Phys. 109, 10350 (1998)

38. M.B. Andersson, J.B.C. Pettersson, Chem. Phys. Lett. 250, 555 (1996)

39. M. van Opbergen, A. Boschetti, S. Iannotta, Opt. Express 4, 53 (1999)

40. K.C. Janda, J.E. Hurst, C.A. Becker, J.P. Cowin, D.J. Auerbach, L. Wharton, J. Chem. Phys. 72, 2403 (1980)

41. K.C. Janda, J.E. Hurst, J.P. Cowin, L. Wharton, D.J. Auerbach, Surf. Sci. 130, 395 (1983)

42. H.Y. Watanabe, H. Yamaguchi, M. Hashinokuchi, K. Sawabe, S. Maruyama, Y. Matsumoto, K. Shobatake, Chem. Phys. Lett. 413, $331(2005)$

43. I. Yasumoto, J. Phys. Chem. 91, 4298 (1987)

44. K.L. Day, Int. Astron. Union Symp. 52, 311 (1973)

45. Y. Matsuo, H. Vach, M. Châtelet, J. Häger, C. Roth, C. Flytzanis, H. Walther, J. Chem. Phys. 93, 4368 (1990)

46. Y. Watanabe, H. Yamaguchi, M. Hashinokuchi, K. Sawabe, S. Maruyama, Y. Matsumoto, K. Shobatake, Eur. Phys. J. D 38, 103 (2006) 